\title{
Scanning Electron Microscopy in Oral Research
}

\author{
Jamaluddin Syed
}

\author{
MDSc, BDS
}

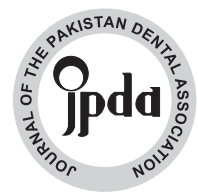

\begin{abstract}
The purpose of this review was to explore the Scanning Electron Microscopy research in latest dental studies done in the recent years. Web search engines Pub med and Google scholar with keywords Scanning Electron Microscopy, sample preparation, scanning electron microscopy detectors were used. The review thus engulfs the sample preparation, types of scanning electron microscopes and briefly about the application of electron microscopy for studying Oral Soft Tissues (Periodontium, Periodontal ligaments, etc.,) and Hard Tissues (Enamel, Dentine). Reliability and authenticity of the Scanning Electron Microscopic analyses was also evaluated in this review. Specimen / Sample preparation methodology were also explored that never been mentioned with clarity in the research publications and comparative analyses was also made of these methods to others.

KEY WORDS: Scanning electron microscopy, sample preparation, dental biomaterials, dental hard tissues, oral soft tissues

HOW TO CITE: Syed J. Scanning Electron Microscopy in Oral Research. J Pak Dent Assoc 2017;26(4):189-195.

DOI: https://doi.org/10.25301/JPDA.264.195

Received: 18 October,2017, Accepted: 11 December, 2017
\end{abstract}

\section{INTRODUCTION}

$\mathrm{H}$ istorically, the word microscope has been taken from ancient Greek words, mikrós and skopeîn, meaning small and to look or see respectively. The science of investigating small objects using such instruments is called Microscopy. ${ }^{1}$ In 1926, foundations of geometrical electron optics were already laid later by Hans Busch, German physicist, when he learnt about the trails of charged particles in electric and magnetic fields. ${ }^{2}$ De Broglie, a French physicist, introduced the concept of corpuscle waves that led to the development of wave electron optics. ${ }^{3}$ Aforementioned discoveries in the electron optics steered towards the concept of electron microscopy. In 1897, Thompson did the discovery of the electron 1926, Busch discovered the Magnetic/Electric Fields as Lenses while at the same year de Broglie revealed the wave nature of the electron. 1931, Max Knoll and Helmut Ruska invented the first 1st Transmission Electron Microscope (TEM). Few years later Erwin Wilhelm Müller, a German physicist, invented the Field Emission Electron Microscope. And in 1938 Manfred von Ardenne built the 1st Scanning Transmission Electron Microscope (STEM). Following year Bodo von Borries and Helmut Ruska introduced the 1st Commercial Transmission Electron Microscope having 10 $\mathrm{nm}$ resolution which improved year by year to $1.0 \mathrm{~nm}$ in 1965 eventually to $0.2 \mathrm{~nm}$. During 1964-1968, Albert Victor Crewe, a British born American physicist, added a field emission gun with the Scanning Transmission Electron

Assistant Professor, Department of Oral Biology, Bhitai Dental and Medical college, Mirpurkhas, Sindh. King Abdul Aziz University, Jeddah, KSA.

Corresponding author: "Dr. Jamaluddin Syed" < drjamalsyed@ gmail.com >
Microscope (STEM) hence raised the resolution further to $\sim 0.3 \mathrm{~nm}$. In 1999 resolution was raised up to < $0.1 \mathrm{~nm}$ and in it came to 2009: $0.05 \mathrm{~nm}^{4,5}$

\subsection{Structure and Working Principle}

Scanning Electron Microscopy (SEM) has been an indispensable tool in research since its invention in $1962^{6}$ and has significantly contributed towards biology, medicine and material sciences research. ${ }^{7-9}$

Electron Microscopes are scientific devices to examine objects on a very fine scale yielding the information about the topography, morphology, composition and crystallographic information. ${ }^{10}$ Electron Microscopy permits the scanning of images at high magnification (50x - 10.000x and above).

Basic construction of an electron microscope involves an Electron Gun, Anode, Electromagnetic lens, Scanning coils, Specimen Holder or Stub, Detectors (SE, BSD, EDS and some more).

A highly energetic beam of electrons is bombarded from the electron gun hitting the sample to produce signals that are eventually collected by a detector SE, BSD, EDS as the case may be to scan the surface of the specimen, Saghiri et al. ${ }^{11}$ In the beginning tungsten material was used to form the tiniest conceivable probe later this source was substituted by LaB6, Transmission Electron and Field Emission guns. The size of the source of the electron is demagnified by the application of electron optics and this is achieved using condenser lenses and an objective lens. By the objective lens the electron beam is focused on the specimen surface. Magnification is limited by a few key parameters: aberrations of the lenses, particularly the objective lens as it works at 
large angles of convergence, the electron source brightness, ${ }^{12}$ and the interaction volume, mainly when the specimens are a bit thick. ${ }^{2}$

\subsection{Electron sources in the electron microscope}

The very initial source for electrons in electron microscopy was a thermionic gun consisting of a V-shaped tungsten filament, having a tip of $100 \mathrm{~mm}$ in radius. When heated with an electric source this tip starts emitting electrons, at temperature of $2700 \mathrm{~K}$ approximately. The thermionic electron source is economical and requires a relatively low vacuum. The tungsten filament is limited to about 100 hours life because of evaporation of its material that makes it thin resulting in its malfunction. The LaB6 gun emitting material, which was introduced later, have a lower work function; therefore the similar amount of electrons can be discharged at lower heating temperatures. For this purpose single-crystal block of LaB6 with a polished tip of $1 \mathrm{~mm}$ radius, about 0.5 $\mathrm{mm}$ long and about $100 \mathrm{~mm}$ in diameter, is used. Subject to the sharpness of the tip, LaB6 gun shows 5-10 times higher brightness and a 10 times longer lifetime when compared to the tungsten filament. ${ }^{2}$ Lately two types of more electron emission methods were introduced namely; cold field emission (CFE), and thermal field emission (TFE). In cold field emission the electrons tunnel through the potential barrier due to formation of a high electric field hence leaving the cathode wire at room temperature. Thermal field emission method operates only at a higher temperature similar in principle to the CFE. This is thought to help in keeping a clean tip and minimizing noise in final results. As the brightness is a key perimeter for the resolution in the SEM defining the current available for a given size of the probe that finally sets the recording time per pixel of the image. This increased brightness is successfully achieved from these field-emission sources having a higher brightness, of the order of $109 \mathrm{~A} / \mathrm{cm} 2 \mathrm{sr}$ at $20 \mathrm{keV} .^{2,13}$ When compared with the thermionic emitters, where the effective source size is of the order of $5 \mathrm{~mm}$, field emission exhibits another advantage as the virtual source is small, usually in the 5-25 $\mathrm{nm}$ range. Lens aberrations are reduced due to lower demagnification and FEG having low energy spread values as low as $0.35 \mathrm{eV}$ for FEG, in comparison with $1.5 \mathrm{eV}$ or more for a thermionic emitter. Chromatic aberration does not essentially limit the resolution at high energies $(>10$ $\mathrm{keV}$ ) and it is a main factor for improvement of low-energy imaging. ${ }^{14}$

\subsection{Signal types in Scanning Electron Microscopy}

The Scanning Electron Microscope provides information by three types of signals Secondary Electrons (SE), back-scattered electrons (BSE) and energy dispersive x-ray analysis (EDX or EDS). ${ }^{15}$ Secondary Electrons (SE) are bombarded on the surface producing an image that can be readily inferred. The sample morphology determines the contrast of that image and therefore a high resolution image is obtained through the help of a small diameter of electron beam. ${ }^{16}$ Back-scattered electrons (BSE) are primary beam of electrons redirected from atoms. The image contrast of a back-scattered micrograph is determined from the atomic number of sample elements. The resolution in back scattered electrons (BSE) image cannot be as good as achieved in secondary electrons (SE). However the image formed shows the distribution of a number of chemical phases. ${ }^{17}$

Mapping of root canal dentin is illustrated in $(B, C)$, $(\mathrm{D}, \mathrm{E})$ and $(\mathrm{F}, \mathrm{G})$ in the coronal, middle and apical portions, indicating some gutta-percha remnants and dentin chips. By the images the differences in both the detectors can be appreciated. Lighter-contrast particles indicate compositional contrast, further confirming that they are gutta-percha and (A) overview of root canal wall. ${ }^{11}$

\subsection{Sample preparation}

The decision should be taken prior whether a sample is suitable to be scanned under Scanning Electron Microscope (SEM). Thickness of a sample not more than $100 \mathrm{~nm}$ is recommended to get best results. Sample preparation can be done by thinning and polishing the materials mechanically. After this there are different ways of placing the samples on the stubs used for scanning under the electron microscope,

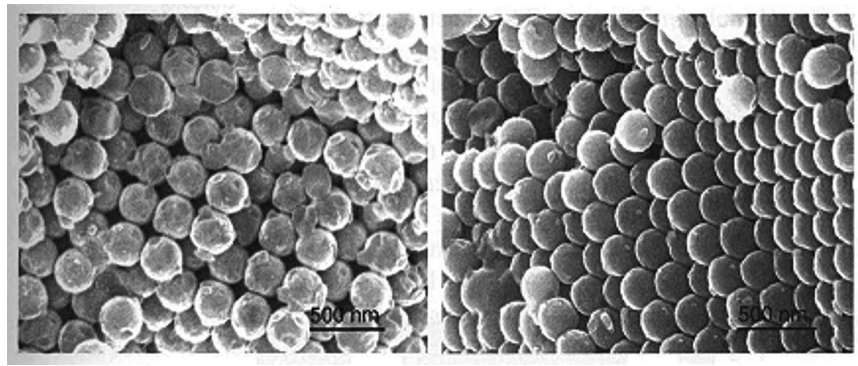

Figure: 1 (Illustration of 1.4.) secondary electron and back scattered electron detectors

either the samples can be glued with some epoxy glue on the sample holder (stub) or can be sticked with Carbon sticking tapes specially designed for the stubs. When a transmission electron microscope (TEM) is used to scan the data is extracted from the margins of a hole in the center of that sample, this hole is made by the ion thinning method, a method in which sample is irradiated with Argon (Ar) ions beams in most instances to create a hole. The eventual damage of ion thinning is reduced by metal coating of the sample before this irradiation. ${ }^{18}$ Therefore scanning with electron microscopy requires care during the sample preparation or else this may affect the analyses and results. 
Saghiri et al. ${ }^{11}$, suggested that cell observation by SEM requires prior application of a fixative like osmium tetroxide and glutaraldehyde. This fixation is generally achieved by incubation in a solution of a buffered chemical, such as glutaraldehyde, which is occasionally combined with formaldehyde and post-fixation with osmium tetroxide remains an option too. Since literature recommends different methodologies, cell adhesion towards surface and other biological interactions might ensue in a different way according to the fixation process. ${ }^{19-21}$ No studies on the subject of comparison of various methods of fixation for specimens/samples evaluated under SEM have been performed till date.

\subsection{Coating Samples}

SEM scans samples that are electrically conductive. For a non-conductive material a special layer or coating of carbon, gold, platinum is applied on the sample to make it conductive and for this a sputtering coating machine is used to serve the purpose.

Few have mentioned the methods for specimen preparation and how the specimens were coated. ${ }^{19,20,22-26}$ Most articles did not even describe the process of controlling the conductivity of the conductive or non-conductive specimens. There is an understanding that different coating methods for the same samples could generate image artifacts. Similarly, the voltage (HV) or the Vacuum conditions used for scanning the images are generally not detailed in the articles. ${ }^{27}$

In SEM conventional imaging, the specimens must be conductive electrically, at least at the surface, and it is made sure that they are electrically grounded to prevent the buildup of electrostatic charge superficially. Apart from cleaning and mounting the specimen on a stub, metals require no specific preparation for SEM. ${ }^{11}$

Non-conductive specimens, like teeth, composites and ceramics likely get charged when scanned under the electron microscope, particularly in Secondary Electron (SE) imaging mode, and this may lead to faults in scanning and presence of other artifacts in imaging, and because of this the samples are generally coated with some electrically-conducting material, as carbon, Gold or platinum, using a low vacuum sputter coating or high vacuum evaporation machine. The accumulation of static electric charge is thus prevented on the specimen for the period of electron irradiation. Saguiri et al. ${ }^{11}$ mentioned two reasons for coating, even when there is enough specimen conductivity to prevent charging: (a) to increase signal and surface resolution, when the samples are of low atomic number (Z); and (b) betterment in the resolution occurs since backscattering and secondary electron emission near the surface are enhanced and therefore a higher-quality image of the surface is achieved.

\section{Dental Applications:}

Since its inception and introduction in dental research electron microscopy has a wide range of applications involving Dental Biomaterials and Dental Hard or soft tissues. 1. Evaluation of micro cracks/gaps and deformities in biomaterials and Dental hard tissues.

2. Details of Surface topography and roughness of different Dental Biomaterials /Dental hard Tissues..

3. Details of Subsurface structures of dental Hard tissues/Dental Biomaterials.

4. Differentiate various dental hard tissue and biomaterial structures.

5. Evaluation of bonds between various biomaterials and structures.

\subsection{Evaluation of Dental Biomaterials:}

a) In one study scanning electron microscopy was applied to evaluate the marginal integrity of bonded resin composite fillings for posterior teeth to enamel and dentine. ${ }^{28}$

b) The purpose of the study was the fabrication of dental glass-ceramics to produce bioactive behaviour around the margins of restorations and to provide a bioactive surface which can lead to attachment of periodontal tissue, providing a complete seal of the marginal gap between tooth and fixed prosthesis. Scanning electron microscope assisted in the establishment of the workability of the new composite material to be applied as coating on the base porcelain as well as the bioactive behaviour of the fabricated coated specimens. ${ }^{29,30}$

c) Development of a novel surface modification for improved bonding to zirconia. Scanning electron microscopy (SEM) were used to evaluate and quantify failure surfaces. ${ }^{31}$

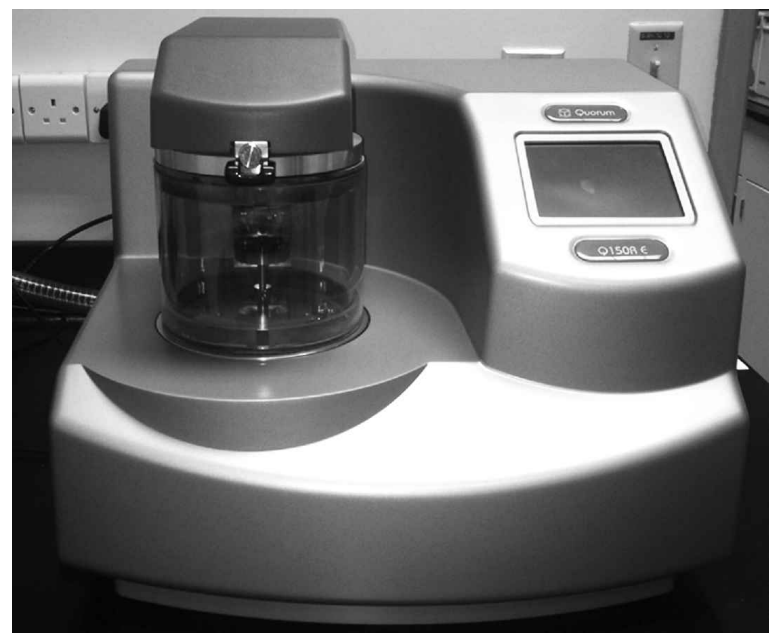

Figure: 2 Illustration of $2.1(\mathrm{a}, \mathrm{b})^{30}$ 

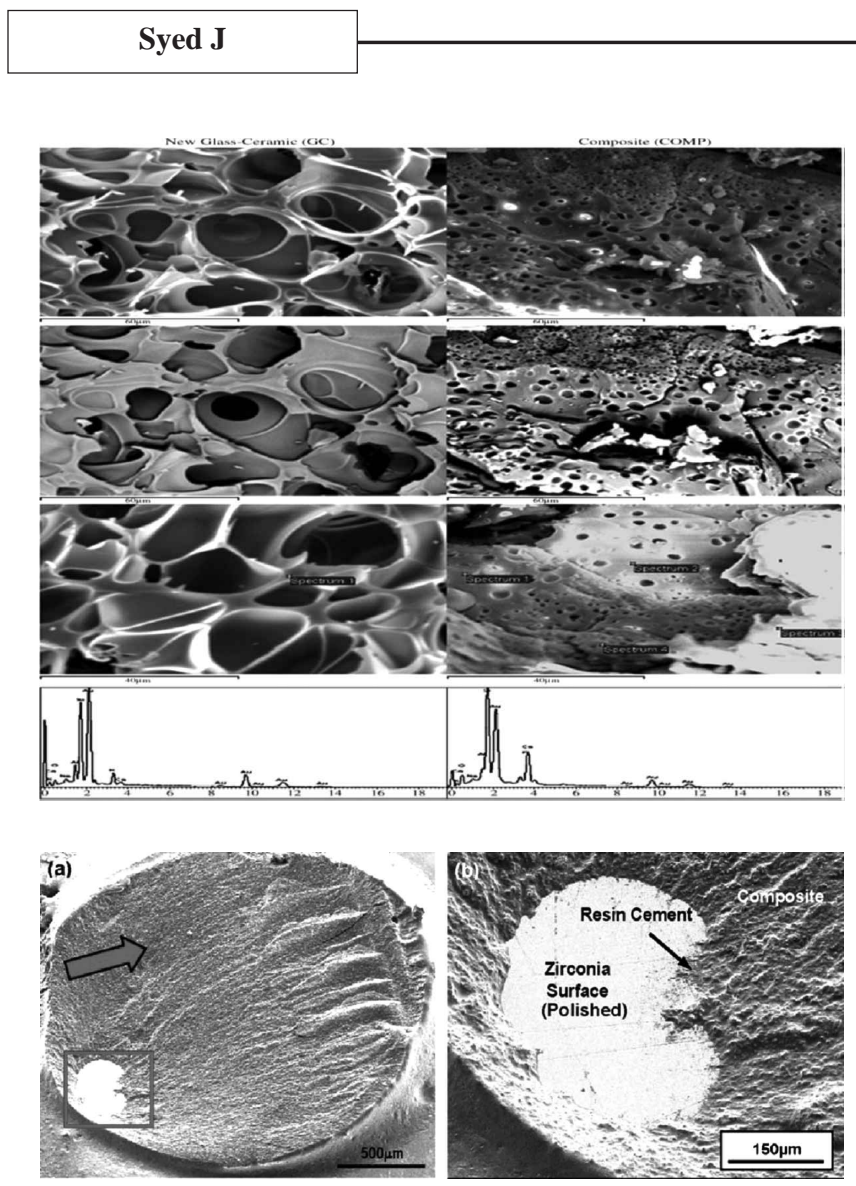

Figure: 3 Showing the sites of the failure and/or improved bonding surfaces 2.1. (c) ${ }^{31}$

\subsection{Dental Hard Tissues:}

a) Methods in microscopic imaging, such as scanning electron microscopy (SEM), ${ }^{32-36}$ transmission electron microscopy (TEM), ${ }^{37-41}$ can also be applied to identify micromorphological alterations at the interface between enamel/dentine and the dental composite biomaterial.

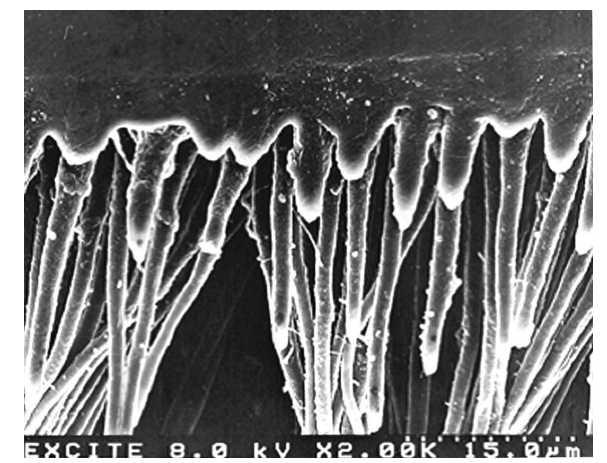

Figure: 4 Image showing classic micromorphological alterations at the interface 2.2 (a).

b) Scanning electron microscopy (SEM) was used for measuring the in vitro resorption of dental hard tissues as one of the earliest techniques ${ }^{31-42}$ and it is still broadly used till date. ${ }^{43-45}$
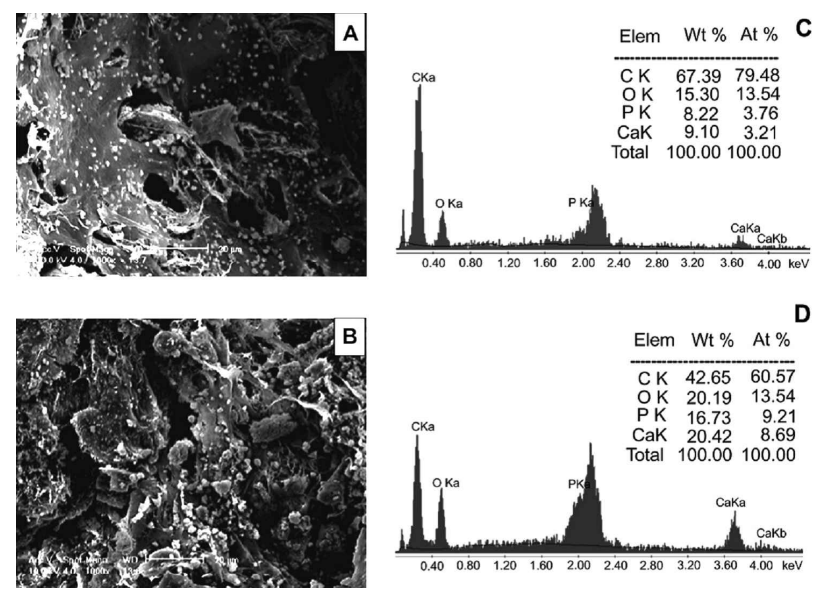

c) Daniela C. Kalthoff in 2011, estimated the microstructure of Dental Hard Tissues in Fossil and Xenarthrans (Mammalia: Folivora and Cingulata) by means of the scanning electron microscopy methods. ${ }^{46}$

\subsection{Oral Soft Tissues:}

a) An appreciable amount of small globular mineral deposits on the surfaces of macropores in solid-walled (SW) poly(1lactic acid) (PLLA) scaffolds was also revealed by SEM images (Fig. A), while an noticeably larger amount of mineral nodules was observed on nanofibrous (NF) poly(l-lactic acid) (PLLA) scaffolds (Fig. B). This observation was confirmed by energy dispersive $\mathrm{x}$-ray microanalysis, which identified higher calcium content $(20.42 \%)$ on the NF scaffolds than on the SW scaffolds $(9.10 \%)$ after 8 weeks of culture. ${ }^{47}$

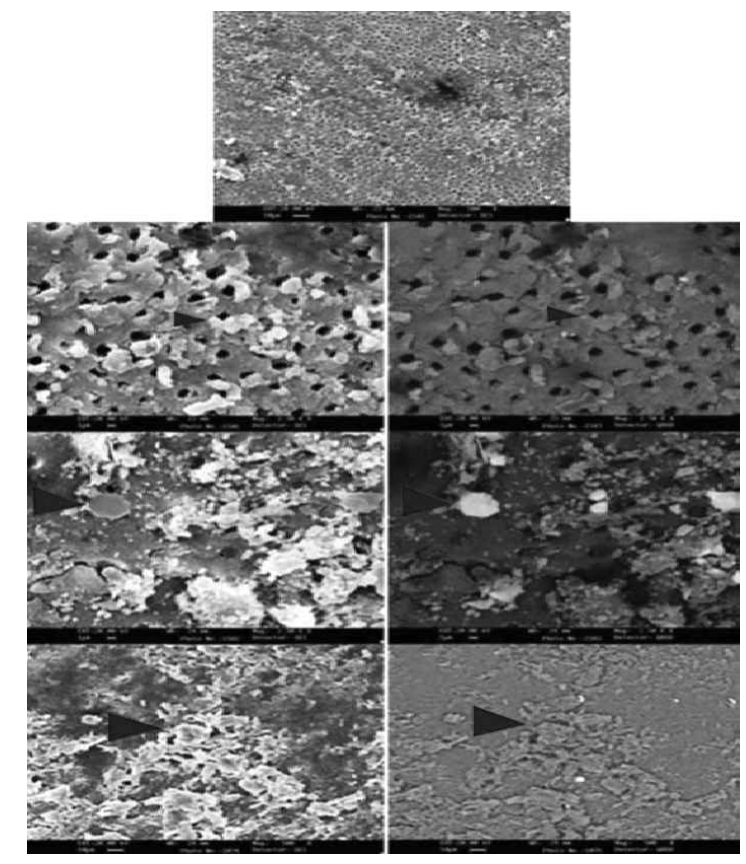

Figure: 5 Illustration of $2.3(\mathrm{a})^{47}$ 


\section{CONCLUSION}

For testing material properties of dental hard or soft tissues it is required to have reliable laboratory techniques. However, the validation and reproducibility of previously available methodologies to assess the materials and dental hard or soft tissues topography and elemental analyses were also useful but the introduction of electron microscopy in the dental research remarkably improved the whole idea.

The SEM technology applied in Endodontics permits visualization of root/dentin structures, with altered heights, without changing the focus. As SEM figures are in gray scale, the color of dentin does not effect in achieving a correct focus, a limitation that researchers use to face in optical stereomicroscopes.

Atomic force microscopy, EDS and X-ray photoelectron spectroscopy are generally associated with SEM topographic examination before and after several treatments..$^{24,48-50}$ SEM techniques are still considered to have limitations to provide indefinable facts for structural features. Researchers in Dentistry should also be cognizant of the guiding principles when thinking about SEM. Imaging principles standardization is equally significant as the research itself. Following the correct guidelines and principles makes easier for readers to comprehend how SEM results were acquired. Therefore, the studies can compare their morphological results, using the similar protocols.

Although SEM is a substantial tool for research in the discipline of dental research, researchers should give comprehensive evidence while using SEM figures, since the assessment of results is likely when same magnifications are used. Moreover, the sample processing, conductivity, type of microscope for instance tungsten, LaB6 beam microscopes or FEG-SEM is such vital information that should also be revealed in the publication. The interpretation and comparison of results becomes difficult for readers when researchers use SEM technology with the lack of the above mentioned information.

\section{CONFLICT OF INTEREST}

None declared

\section{REFERENCES}

1. http://en.wikipedia.org/wiki/Microscope.

2. Bogner A, Jouneau P-H, Thollet G, Basset D, Gauthier C. A history of scanning electron microscopy developments: towards "wet-STEM" imaging. Micron. 2007;38:390-401. 3. Holland PR. The quantum theory of motion: an account of the de Broglie-Bohm causal interpretation of quantum mechanics: Cambridge university press; 1995. 4. Erni et al. Phys.Rev.Lett. 2009, 102, 096101. 5. Zaluzec Introduction to Scanning /Transmission Electron Microscopy and Microanalysis.

6. Stewart AD, Boyde A. Ion etching of dental tissues in a scanning electron microscope. Nature 1962;196:81-2. 7. McComb D, Smith DC. A preliminary scanning electron microscopic study of root canals after endodontic procedures. J Endod 1975; 1:238-42.

8. Yamada RS, Armas A, Goldman M, Lin PS. A scanning electron microscopic comparison of a high volume final flush with several irrigating solutions: part 3. J Endod 1983; 9:137-42.

9. Saleh IM, Ruyter IE, Haapasalo MP, Orstavik D. Adhesion of endodontic sealers: scanning electron microscopy and energy dispersive spectroscopy. J Endod 2003;29:595601.

10. J Goldstein, DE Newbury, P Echlin, DC Joy (Book) Scanning electron microscopy and X-ray microanalysis: a text for biologists, materials scientists, and geologists. 2012 11. Saghiri MA, Asgar K, Lotfi M, Saghiri AM, Neelakantan $P$ et al. Back-scattered and secondary electron images of scanning electron microscopy in dentistry: a new method for surface analysis. Acta Odontol Scand 2012 ;70:603-9. 12. Hawkes, P., Recent advances in electron optics and electron microscopy. Ann. Fondation Louis de Broglie 291. 2004.

13. Pawley, J., 1997. The development of field-emission scanning electron microscopy for imaging biological surfaces. Scanning 19, 324-336.

14. Joy, D.C., 1991. The theory and practice of highresolution scanning electron microscopy. Ultramicroscopy 37, 216-233.

15. Kato M, Homma K, Komura F, Furuya T. Scanning electron microscope. United States patents US Patent 4,803,358.1989.

16. Lloyd GE. Atomic number and crystallographic contrast images with the SEM: a review of backscattered electron techniques. Mineral Mag 1987;51:3-19.

17. Cole DA, Shallenberger JR, Novak SW, Moore RL. $\mathrm{SiO} 2$ thickness determination by $\mathrm{x}$-ray photoelectron spectroscopy, Auger electron spectroscopy, secondary ion mass spectrometry, Rutherford backscattering, transmission electron microscopy, and ellipsometry. J Vac Sci Technol B 2000; 18:440-4.

18. Joachim Mayer, Lucille A. Giannuzzi, Takeo Kamino, and Joseph Michael. TEM Sample Preparation and FIBInduced Damage. Mrs Bulletin, May 2007; volume 32. 19. Coraça-Huber DC, Fille M, Hausdorfer J, Pfaller K, Nogler M. Staphylococcus aureus biofilm formation and 
antibiotic susceptibility tests on polystyrene and metal surfaces. J Appl Microbiol 2012; 112:1235-43. 20. Li HF, Wang YB, Zheng YF, Lin JP. Osteoblast response on Ti- and Zr-based bulk metallic glass surfaces after sand blasting modifi cation. J Biomed Mater Res B Appl Biomater. 2012; 18:1721-8.

21. Rosslenbroich SB, Raschke MJ, Kreis C, Hans-Tholema N, Uekoetter A, Reichelt R et al. Daptomycin: Local Application in Implant-Associated Infection and Complicated Osteomyelitis. ScientificWorldJournal 2012; 18:1-9. 22. Ayobian-Markazi N, Foourutan T, Kharazifar MJ. Comparison of cell viability and morphology of a human osteoblast-like cell line ( $\mathrm{SaOS}-2$ ) seeded on various bone substitute materials: An in vitro study. Dent Res J 2012;9:8692.

23. Ballo AM, Xia W, Palmquist A, Lindahl C, Emanuelsson L, Lausmaa J. Bone tissue reactions to biomimetic ionsubstituted apatite surfaces on titanium implants. J R Soc Interface 2012; 9:1615-24.

24. Barkarmo S, Wennerberg A, Hoffman M, Kjellin P, Breding K, Handa P et al. Nano-hydroxyapatite-coated PEEK implants: A pilot study in rabbit bone. J Biomed Mater Res A. 2012 3:1-7.

25. Bonetti GA, Zanarini M, Incerti Parenti S, Lattuca M, Marchionni S, Gatto MR. Evaluation of enamel surfaces after bracket debonding: an in-vivo study with scanning electron microscopy. Am J Orthod Dentofacial Orthop. 2011;140:696-2.

26. Parangipe A, de Gregorio C, Gonzalez AM, Gomez A, Silva Herzog D, Piña AA et al. Efficacy of the self-adjusting file system on cleaning and shaping oval canals: a microbiological and microscopic evaluation. J Endod 2012;38:226-31.

27. TC Paradella, MA Bottino, Scanning Electron Microscopy in modern dentistry research, Braz Dent Sci 2012;15:43-48. 28. MJ Roggendorf, N Krämer, A Appelt, M Nauman. Marginal quality of flowable 4-mm base vs. conventionally layered resin composite. Journal of Dentistry, 2011;3 9, 6 $43-64$.

29. X Chatzistavrou, D Esteve, E Hatzistavrou. Sol-gel based fabrication of novel glass-ceramics and composites for dental applications. Materials Science and Engineering C, 2010; 30, 730-739.

30. JR Piascik, SD Wolter, BR Stoner . Development of a novel surface modification for improved bonding to zirconia Dental materials, 2011;2 7, e99-e105.

31. J Field, P Waterhouse, M German. Quantifying and qualifying surface changes on dental hard tissues in vitro. Journal of dentistry, 2010;3 8, 182 - 19.

32. Breschi L, Prati C, Gobbi P, Pashley D, Mazzotti G, Teti $\mathrm{G}$, Perdigão J. Immunohistochemical analysis of collagen fibrils within the hybrid layer: a FEISEM study. Oper Dent 2004;29:538-546.

33. Erhardt MC, Osorio R, Pisani-Proenca J, Aguilera FS, Osorio E, Breschi L, Toledano M. Effect of double layering and prolonged application time on MTBS of water/ethanolbased self-etch adhesives to dentin. Oper Dent 2009;34:571577.

34. Erickson RL, Barkmeier WW, Latta MA. The role of etching in bonding to enamel: a comparison of self-etching and etch-and-rinse adhesive systems. Dent Mater 2009; 25:1459-1467.

35. Sato M, Miyazaki M. Comparison of depth of dentin etching and resin infiltration with single-step adhesive systems. J Dent 2005; 33:475- 484.

36. Van Landuyt KL, Cardoso MV, De Munck J, Peumans M, Mine A, Lambrechts P, Van Meerbeek B. Optimization of the concentration of photo-initiator in a one-step self-etch adhesive. Dent Mater 2009;25:982-988.

37. Yiu CK, Hiraishi N, King NM, Tay FR. Effect of dentinal surface preparation on bond strength of self-etching adhesives. J Adhes Dent 2008;10:173-182.

38. Brackett MG, Tay FR, Brackett WW, Dib A, Dipp FA, Mai S, Pashley DH. In-vivo chlorhexidine stabilization of hybrid layers of an acetone-based dentin adhesive. Oper Dent 2009;34:379-383.

39. Breschi L, Mazzoni A, Ruggeri A, Cadenaro M, Di Lenarda R, Dorigo ES. Dental adhesion review: aging and stability of the bonded interface. Dent Mater 2008;24:90101.

40. Mine A, De Munck J, Cardoso MV, Van Landuyt KL, Poitevin A, Kuboki T, Yoshida Y, Suzuki K, Lambrechts P, Van Meerbeek B. Bonding effectiveness of two contemporary self-etch adhesives to enamel and dentin. J Dent 2009;37:872883.

41. Van Meerbeek B, Yoshida Y, Snauwaert J, Hellemans L, Lambrechts P, Vanherle G, Wakasa K, Pashley DH. Hybridization effectiveness of a two-step versus a three-step smear layer removing adhesive system examined correlatively by TEM and AFM. J Adhes Dent 1999;1:723.

42. Boyde A, Lester KS. Electron microscopy of resorbing surfaces of dental hard tissues. Zeitschrift fuer Zellforschung und Mikroskopische Anatomie 1967;83:538-48. 43. Ferrazzano GF, Cantile T, Quarto M, Ingenito A, Chianese L, Addeo F. Protective effect of yogurt extract on dental enamel demineralization in vitro. Australian Dental Journal 2008;53:314-9.

44. Sorvari R, Peltarri A, Meurman JH. Surface ultrastructure of rat molar teeth after experimentally induced erosion and attrition. Caries Research 1996;30:163-8. 45. Schmidlin PR, Gohrinng TN, Schug J, Lutz F. 
Histological, morphological, profilometric and optical changes of human tooth enamel after microabrasion. American Journal of Dentistry 2003;16:4A-8A. 46. Daniela C. Kalthoff, Microstructure of Dental Hard Tissues in Fossil and Recent Xenarthrans (Mammalia: Folivora and Cingulata), Journal of Morphology, 2011;272:641-661.

47. J Wang, H Ma, X Jin, J Hu, X Liu, L Ni, PX Ma. The effect of scaffold architecture on odontogenic differentiation of human dental pulp stem cells. Biomaterials, 2011;32, 7822-7830.

48. Vaquero-Aguilar C, Jimenez-Melendo M, Torres-Lagares
D, Llena-Blasco O, Bruguera A, Llena-Blasco J, GarciaCalderon M, Velazquez-Cayon R, Gutierrez-Perez JL Zirconia implant abutments: microstructural analysis. Int J Oral Maxillofac Implants. 2012; 27:785-91. 49. Fan X, Feng B, Liu Z, Tan J, Zhi W, Lu X et al. TiO2 nanotubes on porous titanium scaffold and biocompatibility evaluation in vitro and in vivo. J Biomed Mater Res A 2012; 13:1-6.

50. Yang FY, Dong W-J, He F-M, Wang X-X, Zhao S-F, Yang, G-L. Osteoblast response to porous titanium surfaces coated with zincsubstituted hydroxyapatite. Oral Surg Oral Med Oral Pathol Oral Radiol 2012; 113: 313-8. 